Check for updates

Cite this: Phys. Chem. Chem. Phys., 2017, 19, 11057

Received 3rd March 2017,

Accepted 31st March 2017

DOI: 10.1039/c7cp01397c

rsc.li/pccp

\section{The effect of chemical ordering and lattice mismatch on structural transitions in phase segregating nanoalloys}

\author{
Kevin Rossi and Francesca Baletto (D) * \\ We elucidate the effect of lattice mismatch and chemical ordering on structural transitions in bimetallic \\ nanoalloys of $\sim 1.5 \mathrm{~nm}$. We show that collective screw dislocation motions happen in small mismatch \\ shellacore systems while strongly mismatched ones favour incomplete outer shell rearrangements. Cooperative \\ transitions can also become hindered when the chemical ordering breaks the geometrical symmetry. Escaping \\ from an unfavourable morphological basin occurs first via re-arrangements of the geometry and then changes \\ towards a better chemical pattern. We observe that the chemical re-ordering mechanisms are independent of \\ system composition and stoichiometry but hinge on the initial and final chemical arrangements.
}

\section{Introduction}

The architecture and chemophysical properties of a nanocluster are intimately related: optical, ${ }^{1,2}$ magnetic, ${ }^{3,4}$ and catalytic ${ }^{5,6}$ features can be tuned by controlling its size, chemical composition and shape, which, in turn, are ruled by the delicate interplay of kinetic, entropic and energetic contributions. ${ }^{7}$

Exquisite control over the size and ordering of a nanoalloy is almost at reach; ${ }^{8-11}$ however, experimental samples are generally polydisperse. ${ }^{7,12}$ Both kinetic and entropic effects ${ }^{13,14}$ contribute to diversify the architectures resulting from gas-condensation, solidification, synthesis or other methodologies. Structural rearrangement and chemical reordering can take place due to ageing, when the nanoalloy is excited by an electron beam, or through thermal annealing. Therefore, it is desirable to predict under which conditions shape fluctuations take place. ${ }^{11,15-17}$

Morphology interconversion processes have been studied for monometallic, core-shell and vertex-decorated systems by means of transition state search algorithms, molecular dynamics or enhanced sampling techniques. ${ }^{18-28}$ It has been shown that solid-solid transitions of cuboctahedral (Co) or decahedral (Dh) morphologies into an icosahedral (Ih) one happen in nanoclusters, with sizes as small as 147 atoms, via cooperative screw-dislocation motions also known as diamondsquare-diamond (DSD) rearrangements. ${ }^{29,30}$ Such a mechanism is the nanoscale equivalent of the bulk martensitic transformation from fcc to bcc. Indeed, both transitions are diffusionless with a lattice deformation driven by a shear motion. The DSD mechanisms lead to a change in the number of twinning planes,

King's College, Physics Department, Strand, WC2R 2LS, London, UK.

E-mail: francesca.baletto@kcl.ac.uk with the transformation of triangular (111) into/from square (100) facets, via a rhombic/diamond (111). ${ }^{26,27}$

A few studies investigated the dynamics of chemical reordering, targeting only subnanometer nanoalloys. ${ }^{31,32}$ They identified two possible rearrangement mechanisms: surface peeling and atomic inter-diffusion. ${ }^{33}$ The former consists of elementary steps of surface diffusion and displacements and leads to segregatedsegregated transformations. The latter comprises vacancy formation and atomic intracluster diffusion and takes place in segregated-mixed transitions.

In this work, we systematically analyse finite temperature solid-solid and chemical reordering in $\mathrm{AgPt}$ and $\mathrm{AgCu}$ nanoclusters with a diameter of $\sim 1.5 \mathrm{~nm}$. In particular, we characterize morphological and chemical rearrangement mechanisms in shell@core and Janus nanoclusters, through a systematic analysis of several different order parameters, which encode detailed information about the structure, chemical species arrangement and transition kinetics. We investigate solid-solid transition mechanisms in $\mathrm{AgPt}$ and $\mathrm{AgCu}$ clusters because of their fascinating catalytic and optical properties and the large number of previous optimization and kinetic studies on them. ${ }^{34-37}$ Our numerical approach offers a fertile perspective to address how mismatch (3\% for $\mathrm{AgPt}$ and $12 \% \mathrm{AgCu}$ ) and chemical ordering influence rearrangements in clusters.

We find that structural transitions among the Ih, Dh, and Co geometries happen in small mismatch shell@core nanoalloys via the same cooperative screw dislocation motions as in their monometallic counterparts. On the other hand, these mechanisms are hindered in strongly mismatched systems. They might also be altered in systems where the chemical ordering violates the geometrical symmetry: starting from an unfavourable shape with a Janus chemical ordering, for example, the rearrangements are 
constrained by the Janus cut. We further note that morphological changes always precede chemical reordering in systems presenting both unfavourable geometries and chemical patterns. Finally, regardless of the initial shape and mismatch of the nanoalloy, we show that surface diffusion happens during segregated to segregated phase transitions, while atomic intercluster diffusion drives the chemical reordering processes of mixed phases.

\section{Models and methods}

The considered architectures are reported in Fig. 1, where "@”" stands for a core@shell ordering and "||" is used for referring to a Janus pattern. We fix the size at 147 atoms and choose to focus on the structural changes and chemical reshuffle of a silver outer shell over a $\mathrm{Cu}$ or Pt core, likely to be the favourable chemical ordering ${ }^{36,37}$ and a Janus order with a cut along the (100) facet of a Co and perpedicular to the five-fold axis for a Dh. In the case of a Janus architecture, we consider a $3: 2$ stoichiometry. The rationale behind our system's choice is to assess the mismatch effect in the case of both spherical and non-spherical chemical patterns and to address the competition between structural and chemical rearrangement mechanisms. We focus on the 147-atom sizes because pure Ag, Pt and $\mathrm{Cu}$ nanoparticles show DSD mechanisms at this size, while intra-structural basin transitions can take place at larger sizes. ${ }^{38}$

To investigate structural transitions in bimetallic nanoalloys we couple molecular dynamics (MD) with a metadynamics (MetaD) algorithm or an iterative temperature scheme (itMD). Newton's equations of motion are solved using a velocity-Verlet algorithm with a time step of $5 \mathrm{fs}$ while an Andersen thermostat regulates the temperature of the system. Metal-metal interactions are modelled within the second moment tight binding approximation $^{39}$ potential and the employed parametrization follows the literature. ${ }^{36,37}$

ItMD consists of a series of concatenated MD runs where the temperature is slowly increased at a constant rate. Here, we leave the system free to move for at least $1 \mathrm{~ns}$ before the temperature is increased to $50 \mathrm{~K}$. We start from tempeartures as low as $100 \mathrm{~K}$ up to the melting transition. The 1 ns time frame should be

Pt55@Ag92
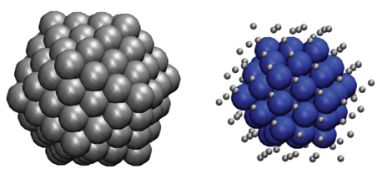

Cu55@Ag92
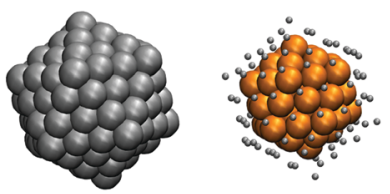

Fig. 1 AgPt (top) and $\mathrm{AgCu}$ (bottom) nanoalloys: an Ih with a core@Ag-shell and the view of the 55-atom inner core; Janus-Dh cut perpendicularly to the 5 -fold axis (middle), and a Janus-Co cut along a (100) plane (right). Ag, Pt, and $\mathrm{Cu}$ atoms are coloured in silver, blue, and orange, respectively. sufficiently long to observe structural changes and to characterise the appearance of any martensitic transition. ${ }^{40}$ We run at least three independent simulations for each initial system.

MetaD exploits a history dependent potential, built by summing Gaussians in a collective variable (CV) space, to enhance the exploration of the conformational space of the system and eventually reconstruct its free energy. ${ }^{41}$ As discussed in our recent work, ${ }^{25}$ we can use two window functions (WF), set at characteristic distances of the pair distance distribution function (PDDF), to investigate structural transitions in nanoclusters. Those CVs are evaluated as:

$$
\mathrm{WF}=\sum_{i, j ; i \neq j} \frac{1-\left(\frac{r_{i j}-d_{0}}{r_{0}}\right)^{6}}{1-\left(\frac{r_{i j}-d_{0}}{r_{0}}\right)^{12}},
$$

with $r_{i j}$ being the distance between the $i$ and $j$ atoms, $r_{0}$ the window width, and $d_{0}$ the characteristic position. In bimetallic systems, depending on the chemical type of the $i$ and $j$ atoms, we consider the corresponding bulk lattice parameter or, for hetero-pairs, their average. The first window function (CV1) is set at the characteristic hcp peak $(\sqrt{8} / 3)$ to count the stacking fault number and to enhance sliding and rotation of the (111) planes; the second one (CV2), positioned at around the 5th neighbour distance, is correlated with altered breathing modes and sliding mechanisms. For this choice of CVs, the MetaD potential evolves every 10 ps by the deposition of a Gaussian of height $0.15 \mathrm{eV}$ with a system dependent width, in the 20-30 range. These two collective variables distinguish among different shapes but they are little sensitive to the chemical ordering. As a paradigmatic example, we compare three points in the (CV1, CV2) plane, namely, (1025, 1204), (956, 1155), and (630, 720), corresponding to Ag82\|Cu65 Ih, Ag92@Cu55 Ih and Ag92@Cu55 Co, respectively. Clearly, the last two points, with the same chemical ordering but different shapes, are far apart, while the first two, with the same shape but different chemical patterns, are much closer, and within a few Gaussians. More importantly, these collective varibles poorly boost any movements via single atomic diffusion, which is the elementary step by which chemical reordering results. ${ }^{33}$ As the coordination number is a robust $\mathrm{CV}$ to drive the latter mechanism, ${ }^{42,43}$ we propose the hetero (heCN) and homo (hoCN) coordination numbers as efficient CVs to simulate chemical reordering via single atom intracluster or surface diffusion. Taking $p_{0}$ as the nearest neighbour bulk reference distance, $q_{0}$ as the distance related to the width of the descending branch of a sigmoid function $f$, and $n$ and $m$ as the powers used to tune smoothness and asymptotic behaviour of the analytic function $f$, the hetero coordination number, HeCN, results:

$$
\begin{gathered}
\operatorname{HeCN}=\sum_{i, j} f\left(r_{i j}\right), \\
f\left(r_{i j}\right)= \begin{cases}1 & \text { if } r_{i j} \leq p_{0}, \\
\frac{1-\left(\frac{r_{i j}-p_{0}}{q_{0}}\right)^{n}}{1-\left(\frac{r_{i j}-p_{0}}{q_{0}}\right)^{m}} & \text { if } r_{i j}>p_{0},\end{cases}
\end{gathered}
$$


The same analytical formulation, with an appropriate choice for $p_{0}$, holds for the homo-atomic coordination, HoCN. The Janus and shell@core structures are well separated along the HeCN collective variable, the latter being at 476, for $\mathrm{Ag}_{92} @ \mathrm{Pt}_{55}$, and the former at 253, in the case of $\mathrm{Ag}_{86} \| \mathrm{Pt}_{61}$. When $\mathrm{HeCN}(\mathrm{Ag}-\mathrm{M}$, $\mathbf{M}=\mathrm{Pt}, \mathrm{Cu}$ ) is employed as a unique $\mathrm{CV}$, the Gaussians are deposited every $10 \mathrm{ps}$, are $0.1 \mathrm{eV}$ tall and 2 (adimensional) wide. Although using HeCN as sole collective variables enables efficient sampling of minima with various chemical ordering and energy differences of several $\mathrm{eV}$, it does not encode enough information to discriminate among nanoparticle shapes. This implies that clusters presenting different motifs but similar chemical ordering are projected onto the same basin, hampering the possibility of correctly reconstructing the free energy profile.

\subsection{Architecture and kinetics characterization}

The nanoalloy morphology is monitored by means of the Common Neighbour Analysis (CNA) ${ }^{44}$ which characterizes the local connectivity around each pair of nearest neighbours in the system. It associates with them a signature $(r, s, t)$ dependent on the connectivity of their neighbourhood: $r$ is the number of their common neighbours, $s$ is the number of bonds between the $r$ atoms, and $t$ is the longest chain among them. The $(4,2,2)$ CNA signature is assigned to pairs along grain boundaries and is used here to distinguish among different morphologies.

The degree of mixing between the chemical species is probed by the segregation parameter $\mu,{ }^{45}$ defined as:

$$
\mu=\frac{\mathrm{HoCN}-\mathrm{HeCN}}{\mathrm{HoCN}+\mathrm{HeCN}}
$$

$\mu$ takes a value of 1 for fully segregated systems and -1 for fully ordered mixed systems. Phase segregated or mixed arrangements are respectively identified by a positive or a negative mixing parameter value.

The spatial distribution of the two chemical species is monitored looking at the evolution of the chemical radius of gyration, $G^{\alpha}$ :

$$
G^{\alpha}=\sqrt{\frac{1}{N^{\alpha}} \sum_{i \in \alpha}\left(r_{\mathrm{com}_{i}}\right)^{2}}
$$

where $r_{\text {com }_{i}}$ is the distance of atom $i$ from the center of mass of the whole cluster, and the sum is over $N^{\alpha}$ atoms of the $\alpha$ chemical species. ${ }^{31}$ In shell@core arrangements, $G^{\mathrm{Ag}}$ and $G^{\mathrm{M}}$, with $\mathrm{M}=\mathrm{Cu}$ or $\mathrm{Pt}$, assume two values corresponding to the two layers around the centre of mass populated by $\mathrm{Ag}$ and $\mathrm{M}$-atoms, respectively, while the Janus ordering, with a roughly 1:1 composition, is characterised by a single value similar to both species.

To provide an estimate of the mobility of a chemical species during a reordering process, we contrast the nearest neighbour adjacency matrix of the system, $M_{i j}(t)$, at two different time steps $t$ and $t+\Delta t . M_{i j}(t)$ is an $N$ by $N$ matrix, with $N$ being the total number of atoms in the system, with entries equal to 1 or 0 depending on whether the distance between atom $i$ and atom $j$ falls below or above the nearest neighbour distance. The latter is set at the intermediate distance between the first two peaks of the pair distance distribution function of the $\alpha$ chemical species. The overall mobility of a chemical species during a motion of the cluster can be estimated by summing the index of atomic homo-rearrangements, $R_{i}^{\alpha}$ :

$$
R^{\alpha}(t, t+\Delta t)=\sum_{i \in \alpha, j \neq i} R_{i}^{\alpha}(t, t+\Delta t)
$$

where $R_{i}^{\alpha}$ is given by

$$
R_{i}^{\alpha}(t, t+\Delta t)=\sum_{i, j \in \alpha, j \neq i}\left|M_{i j}^{\alpha, \alpha}(t+\Delta t)-M_{i j}^{\alpha, \alpha}(t)\right| .
$$

No structural or chemical ordering changes around atom $i$ correspond to a $R_{i}^{\alpha}(t, t+\Delta t)=0$, while a full rearrangement in the neighbourhood of an fcc bulk atom within a time step $\Delta t$ instead leads to $R_{i}^{\alpha}(t, t+\Delta t)=24$, twice the bulk nearest neighbours.

To quickly monitor the starting of a chemical reshuffling, a useful quantity is the ratio of atoms of species $\alpha$ which change at least one of their first neighbours, $H^{\alpha}$,

$$
H^{\alpha}=\sum_{i \in \alpha}^{N^{\alpha}} \frac{\Theta\left(R_{i}^{\alpha}(t, t+\Delta t)\right)}{N^{\alpha}}\left\{\begin{array}{l}
\Theta(0)=0 \\
\Theta\left(R_{i}^{\alpha}\right)=1 \forall R_{i}^{\alpha}>0
\end{array} .\right.
$$

$H^{\alpha}$ ranges between $[0,1], H^{\alpha}=0$ when no homo-atomic pair has changed, $H^{\alpha}=1$ when every $\alpha$ atom has at least one change in its neighbourhood, within a time step $\Delta t$. We give here a final comment on the time-scale over which structural transitions should be monitored. As a typical period for adatom diffusion on a flat surface is expected to be close to $10 \mathrm{ps}$, we choose $\Delta t$ equal to $10 \mathrm{ps}$, as self-adatom diffusion is the fastest rearrangement process we would like to monitor.

\section{Morphological rearrangements: results and discussion}

\subsection{Mismatch effect}

The mismatch between chemical species in the nanoalloy affects the rearrangement kinetics of solid-solid transitions among closed-shell polyhedra, closed polyhedra in the $\mathrm{Pt}_{55} @ \mathrm{Ag}_{92}$ and $\mathrm{Cu}_{55} @ \mathrm{Ag}_{92}$, as highlighted by changes in the $(4,2,2)$ CNA signature for the whole cluster and the inner core, see Fig. 2. Let us first discuss the reconstruction in a small mismatch alloy, $\mathrm{Pt}_{55} @ \mathrm{Ag}_{92}$. Ih $\leftrightarrow$ Co and $\mathrm{Ih} \leftrightarrow$ Dh transitions via the DSD mechanism happen simultaneously in the inner Ptcore and in the Ag outer shell, and they proceed exactly as in the case of monometallic systems. ${ }^{25}$ The (100) facets of Dh or Co first deform into a (111) diamond, which after a collective rotation of the atoms in the cluster becomes two distinct and adjoint (111) facets of an Ih morphology. The inverse process, although more energetically expensive, is also feasible. The free energy barrier for the $\mathrm{Co} \rightarrow \mathrm{Ih}(\mathrm{Co} \leftarrow \mathrm{Ih})$ transition is $0.25 \mathrm{eV}$ (3.6 eV). A partial overlap between the Ih and Dh conformational basins appears due to the sampling of intermediate defected structures; we heuristically determine an upper bound on the barriers dividing the two geometries, $1.5 \mathrm{eV}(4.3 \mathrm{eV})$ for 
Ih

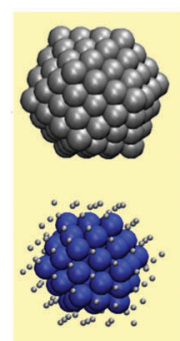

Co

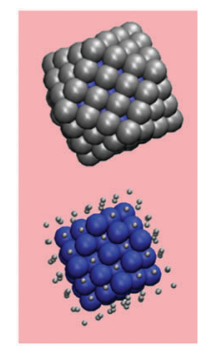

42
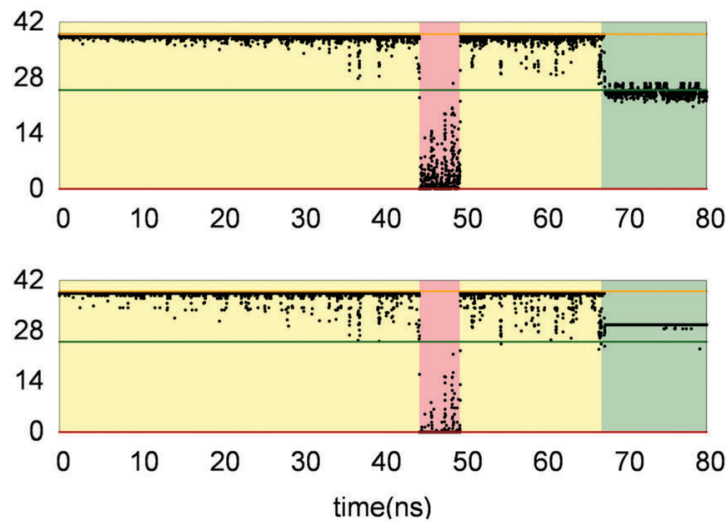

$\mathrm{Dh}$

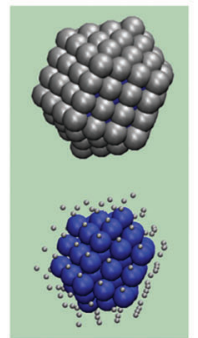

Ih

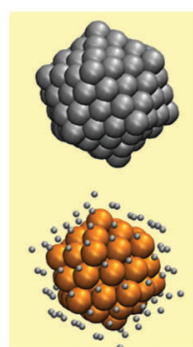

Co@Ih Dh@Ih
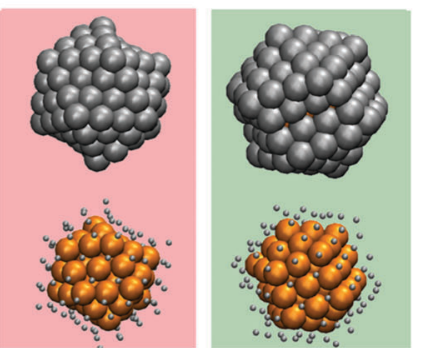
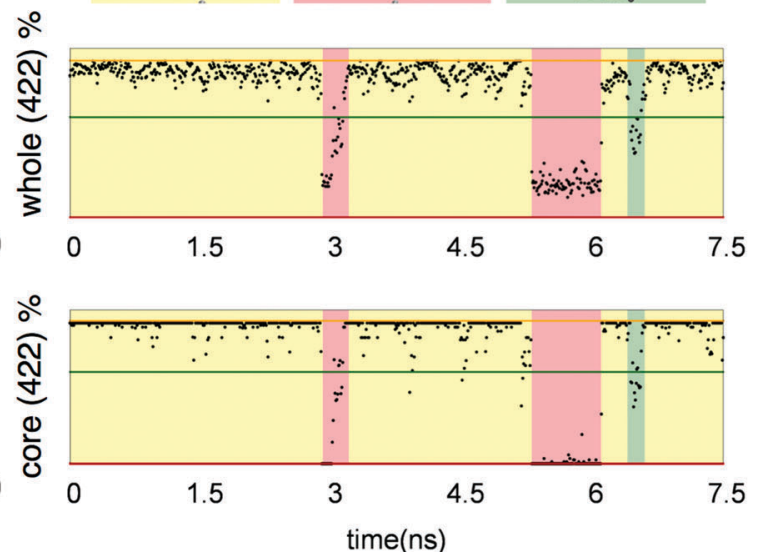

Fig. 2 (422) CNA signature, in \%, of the whole cluster (top) and the 55-atom core (bottom) for $\mathrm{Pt}_{55} \mathrm{aAg}_{92}$ (right) and $\mathrm{Cu}_{55} \mathrm{QAg}_{92}$ (left) during the course of MetaD runs at $300 \mathrm{~K}$. Reference values for ideal Ih, Dh, and Co geometries are shown via straight gold, green and red lines. When Ih, Dh and Co geometries are sampled, the plot is highlighted in yellow, dark-green or pink. A representative snapshot for each of the above mentioned geometries is reported. Colour coding of atoms follows that in Fig. 1.

the $\mathrm{Dh} \rightarrow \mathrm{Ih}(\mathrm{Ih} \leftarrow \mathrm{Dh})$ transition, by summing up the amount of MetaD potential needed to escape the initial Dh (Ih) basin.

A different mechanism is observed in the case of a large mismatch alloy, $\mathrm{Cu}_{55} @ \mathrm{Ag}_{92}$. In this case, the geometric constraints imposed by the mismatch undermine the completion of the DSD rearrangement in the cluster outer shell. As shown in Fig. 2, the $(4,2,2)$ CNA signature of the whole cluster does not correspond to the ones for perfect $\mathrm{Dh}$ and Co clusters of 147 atoms, yet the $\mathrm{Cu}_{55}$ core assumes a $\mathrm{Dh}$ and a Co geometry. The $\mathrm{Cu}$ core undergoes a clear $\mathrm{Ih} \rightarrow \mathrm{Dh}$ or $\mathrm{Ih} \rightarrow$ Co transition following a DSD mechanism, but this movement is hindered for the Ag external shell which displays 5-fold twinning and distorted and concave (111) facets, reminiscent of a non-perfect chiral shell. ${ }^{46}$ This novel family of rearrangement mechanisms resembles the shell-wise Mackay transformations first reported in $\mathrm{Fe}_{561}$ large-scale DFT optimization. ${ }^{47}$ The thus sampled Dh@Ih and Co@Ih structures are a few eV more favourable than the perfect $\mathrm{Dh}$ and Co structures. We notice that the $\mathrm{Cu}$ inner core still performs a DSD movement with a barrier as low as $0.1 \mathrm{eV}$, similarly to the one found in the case of pure $\mathrm{Cu}_{55}{ }^{38}$ The fact that the $\mathrm{Cu}_{55}$-core has a similar barrier in the gas phase and is covered by an Ag outer shell suggests a weak interaction between the two parts of the clusters.

\subsection{Chemical ordering effect}

In Fig. 3, we report two paradigmatic examples of the transition from Janus Co and Dh geometries towards a Janus Ih, in the case of $\mathrm{AgCu}$. We stress from the beginning that the rearrangement mechanisms are the same as in AgPt clusters, showing a negligible role of the mismatch in this case. We observe that geometrical reconstructions towards more energetically favourable structures, such as the Ih geometry, always precede chemical reordering. In addition, we find that these geometrical transitions are martensitic and happen below room temperature. The DSD movement in the Co $\rightarrow$ Ih transition happens by the twisting and stretching of the square facets, around an axis which goes through the center of the triangular facets. The Janus cut in the initial Co geometry is parallel to two square (100) facets and perpendicular to the other two, while it discriminates triangular (111) facets in pure $\mathrm{Ag}$ and $\mathrm{Cu}$ rich clusters. The latter presents either $\mathrm{Ag}$ edge or one $\mathrm{Ag}$ vertex. The DSD mechanism leads to the splitting of the two mixed square (100) facets into four mixed triangular (111) facets.

In the case of Dh, we notice a new mechanism. The Janus cut is along the (100) interface, perpendicular to the 5 -fold axis. The axis is maintained during the transformation towards an Ih geometry. However, the rearrangement is not any longer driven by the single-step DSD mechanism, but consist of two-steps: the formation of a mis-stacked Ih\|Ih morphology, followed by the rotation of its two halves to form a perfect Ih geometry. Indeed, the two halves do a separate DSD motion along the same 5 -fold axis but rotating in opposite directions resulting first in a (100) interface between silver and copper regions. A final rotation of one species with respect to the other completes the formation of a Janus Ih. 

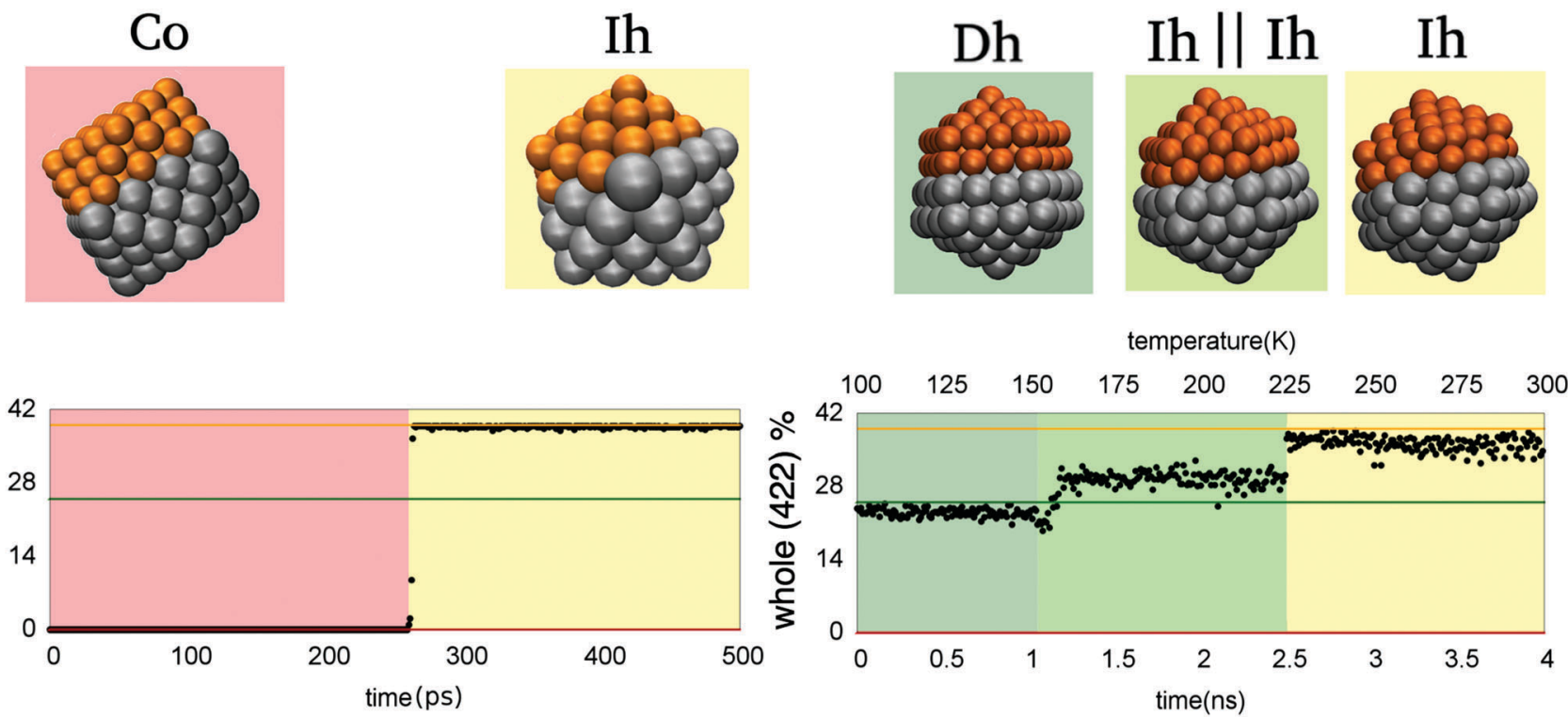

Fig. 3 Structural transition in Janus nanoalloys: (422) CNA signature percentage for the Co $\rightarrow$ Ih transition in $\mathrm{Ag}_{82} \| \mathrm{Cu}_{65}$ during a MetaD run at $10 \mathrm{~K}$ (left); a paradigmatic itMD snapshot for the $\mathrm{Dh} \rightarrow$ Ih transformation in $\mathrm{Ag}_{86} \| \mathrm{Cu}_{61}$, where the appearance of the Ih\|lh motif is highlighted by a light-green window (right). The colouring scheme is the same as in Fig. 2.

We would like to stress that below $500 \mathrm{~K}$, on a hundreds of ns timescale, no chemical changes take place on both $\mathrm{AgCu}$ and AgPt nanoalloys with an initial Janus pattern, although it is energetically unfavorable. Indeed, the melting transition is contemporary or next to the appearance of the shell@core pattern. To define the melting point, in addition to the CNA analysis, we monitor the vanishing of the second peak of the pair distribution function. We would like to note that an unfavourable chemical pattern may lower the melting point and that a non-linear dependence against stoichiometry appears. Referring to the $\mathrm{Ag}-\mathrm{Ih}_{147}$ melting point of $660 \mathrm{~K}$, a single $\mathrm{Cu}$ impurity substitution at the Ih centre shows a $20-25 \mathrm{~K}$ increment, ${ }^{48}$ while the Ag-shell@Cu-core ${ }_{55}$ melts at $650 \mathrm{~K}$ and pure $\mathrm{Cu}-\mathrm{Ih}_{147}$ close to $750 \mathrm{~K}$. Focusing on the $\mathrm{Ag} @ \mathrm{Cu}$ case, the occurrence of the first hetero neighbour pairs increases and peaks to a value corresponding to the $\mathrm{Ag} / \mathrm{Cu}$ interface radial distance suggesting the formation of a shell@core at $575 \pm 25 \mathrm{~K}$, and also suggesting that the Janus $\rightarrow$ shell@core reordering can take place via solid or liquid state in the 575-650 K temperature range.

\subsection{Chemical reordering mechanisms}

To show that the sampled initial and final chemical orders determine whether surface adatom diffusion or intershell diffusion takes place, with a little influence of the mismatch and initial geometry, let us analyse the paradigmatic case of structural and chemical evolution in $\mathrm{Dh}^{\mathrm{Ag}_{86}} \| \mathrm{Cu}_{61}$ via an itMD procedure. Fig. 4 reports the evolution of the $(4,2,2)$ CNA signature, the chemical indexes, segregation parameter $\mu$ and chemical radius of gyration $G^{\alpha}$, as well as the evolution of $H^{\alpha}$ and $R^{\alpha}$ to detail the kinetics of the reorganization. After the first $3 \mathrm{~ns}$ we observe the aforementioned transition from Dh to Ih through the Ih\|Ih configuration where only a Cu-layer rotates, followed by the Ag-region which finalises the rotation towards the Janus-Ih, Fig. 3. The Janus-Ih results to be a long-lived metastable one surviving up to $450 \mathrm{~K}$. At this temperature, the $\mathrm{Ag}$ half starts to disorder and surface defects appear and surface diffusion onto the (111) facets starts. This is consistent with the diffusion barrier of a $\mathrm{Ag}$ adatom jumping onto an $\mathrm{Ih}$ copper cluster. ${ }^{49,50}$ Just below $600 \mathrm{~K}$, the chemical reordering from the defected Janus cluster to a defected shell@core arrangement takes place via several atomic surface diffusion and inter-cluster rearrangements. In the initial steps of the reordering process, the mobility of Ag atoms is larger with respect to $\mathrm{Cu}$, both in terms of the number of $\mathrm{Ag}$ atoms modifying their connectivity and the number of bonds changed. Above $600 \mathrm{~K}$, in agreement with recent free energy calculations ${ }^{51}$ a shell@core pattern is formed and maintained at least up to $750 \mathrm{~K}$, with both $\mathrm{Ag}$ and $\mathrm{Cu}$ halves very mobile and undergoing several structural fluctuations.

Thanks to the introduction of $\mathrm{HeCN}$ as a unique collective variable, chemical reordering transitions can be sampled by means of Metadynamics. As a paradigmatic example, in Fig. 5 we report the evolution of the chemical indexes $\mu$ and $G^{\alpha}$ during a $200 \mathrm{~ns}$ run for $\mathrm{Pt}_{55} @ \mathrm{Ag}_{92}$, where the initial configuration is a perfect core@shell Ih. We observe reordering in the inner shells of the cluster, as signalled by the increasing radius of gyration in Pt and the decrease of the segregation parameter, corresponding to an increase of the number of hetero bonds. During such rearrangements, the outer shell is always Ag-rich, if not made only of Ag atoms, while the two inner shells are Pt-rich; occasionally also an Ag@Pt@Ag multi-shell ordering is sampled, in the 140-160 ns time interval. Only after $170 \mathrm{~ns}$, in correspondence with a similar radius of gyration for $\mathrm{Ag}$ and $\mathrm{Pt}$, and an increase in the segregation parameter, do we observe the formation of a Janus pattern. Looking at the details of the mechanisms, our simulations show that the core@shell $\leftrightarrow$ Janus reordering transition happens 


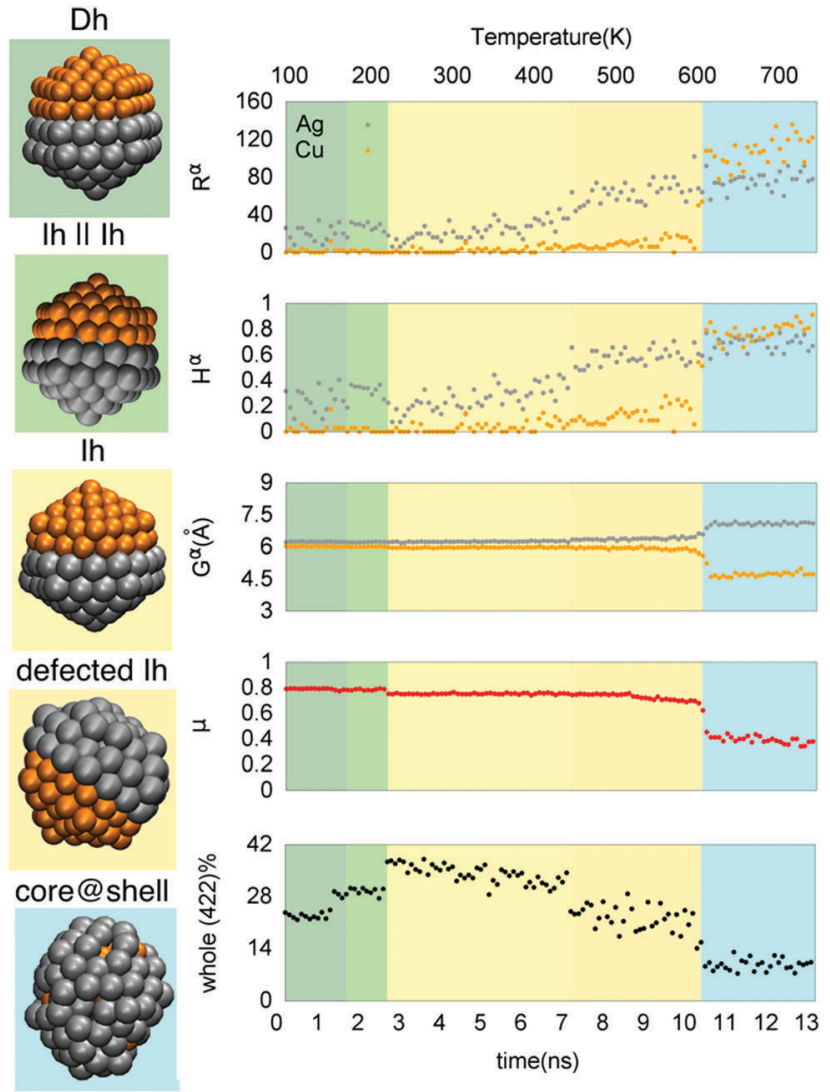

Fig. 4 Typical snaptshots during the itMD dynamics of $\mathrm{Ag}_{85} \mathrm{Cu}_{62}$ Dh up to the melting point. Five regions are found, shadowed by different colours, in correspondence with changes in the $(4,2,2) \% . \mu$ and $G^{\alpha}$ show that the Janus pattern is altered after the formation of an Ih morphology, but a core-shell is completely formed only after $600 \mathrm{~K}$, very close to, if not after, the melting point. From the evolution of the $R^{\alpha}$ and $H^{\alpha}$ parameters, Ag (grey dots) is initially more mobile than $\mathrm{Cu}$ (orange). Ag starts to move clearly after $450 \mathrm{~K}$ with the formation of a defected Ih in the silver half, while Cu atoms start to move above $550 \mathrm{~K}$.
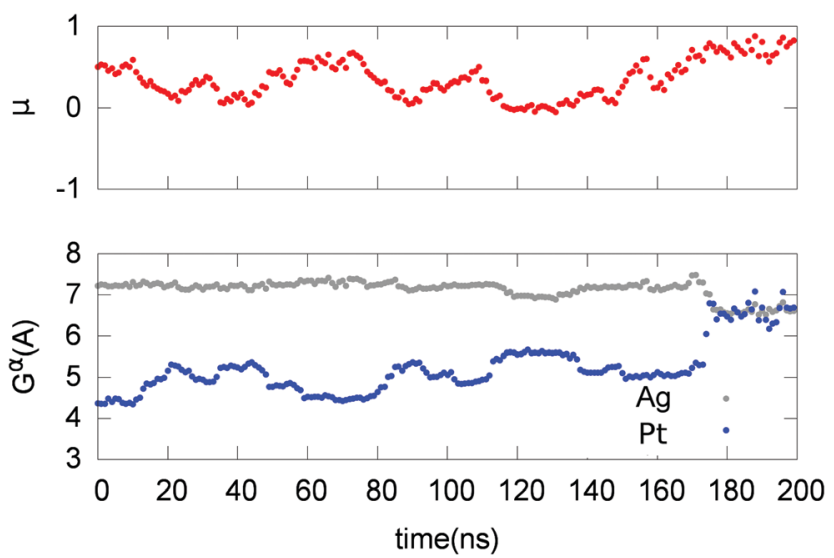

Fig. 5 Evolution of the chemical radius of gyration $\left(G^{\alpha}\right)$ and of the segregation parameter $(\mu)$ for $\mathrm{Ag}$ (silver) and $\mathrm{Pt}$ (blue), during a $\mathrm{HeCN}$ driven MetaD run at $300 \mathrm{~K}$ starting from $\mathrm{Ih} \mathrm{Pt}_{55} \mathrm{AAg}_{92}$. The icosahedral shape is maintained during the evolution.

via surface adatom diffusion while random mixing $\leftrightarrow$ core@shell takes place via vacancy formation and intracluster atomic diffusion, in agreement with previous studies. ${ }^{31}$ These two atomic motions can happen simultaneously and lead to a variety of reordering pathways which connect, for example, mixed to Janus arrangements or multi-shell to core-shell patterns.

\section{Conclusion}

We show a rich panorama of rearrangement mechanisms in Ag-nanoalloys with sizes as small as 147 atoms by means of two enhanced sampling techniques based on molecular dynamics. We address how chemical ordering and mismatch affect structural transition pathways among different morphologies with a $\mathrm{Ag}$ fraction of $59 \pm 3 \%$. We would stress that the stoichiometry can influence the structural reorganisation pathway, as shown for $\mathrm{AuAg}_{147}{ }^{28}$ and for $\mathrm{AgCu}$ and $\mathrm{AgAu}$ nanoalloys, ${ }^{51}$ but a systematic study at different compositions was out of the scope of this work, and we focused on addressing the mismatch effect on structural and chemical transitions. To sample chemical reorderings more efficiently, we introduce the hetero-atomic coordination number as a sole collective variable of a Metadynamics framework because of its ability to enhance both surface and intra-cluster diffusion. We remark that the chemical coordination numbers are order parameters which may be easily transferable to boost the sampling of a variety of systems where (de)alloying processes or mixing-segregation transitions are of interest. We propose new and proper descriptors to characterise the geometry, chemical mixing, and mobility of the two chemical species during the solid to solid transformations. Those quantities are based on common neighbour analysis and evolution of the cluster adjacency matrix. Therefore they can be applied to characterise the dynamics of other nanosystems.

We observe that the diamond-square-diamond rearrangement ${ }^{29}$ connects Ih to Co and Dh morphologies in core@shell nanoalloys when the mismatch is less than $10 \%$. In contrast, an incomplete diamond-square-diamond rearrangement, similar to the shell-wise Mackay ${ }^{47}$ mechanism, takes place in a system characterised by a larger mismatch, where only the inner core can undergo a complete transformation. Structural transitions in Janus nanoalloys are shape dependent: while Co rearranges into Ih via the DSD mechanism, this cooperative screw dislocation motion is hindered in Dh because it is energetically favourable to have the formation of a (111) interface. This happens throughout a rotation around the 5-fold axis in the opposite direction in the two chemical species, leading to the formation of an Ih\|Ih motif and then a Janus-Ih. The latter is stable up to $450 \mathrm{~K}$, when the Ag-adatom diffusion on (111) starts to be thermally activated. In contrast to morphological rearrangements, which involve rotations, screw and concerted motion, chemical reordering takes place via surface and/or intra-cluster diffusion regardless of the system's composition. We note that a metastable shape first underwent a morphological transition towards a favourable geometry and only then does the chemical reordering start. We want to stress that some chemical patterns make escaping from the Ih geometry very unlikely, as for example the Janus-Ih which is a basin of attraction, although energetically unfavourable. This is a quite important result that needs to be exploited as there is a chance 
to tune shape transitions by playing with the initial chemical ordering. For the considered Ag-nanoalloys, a Janus motif will evolve towards a Janus-Ih in a wide temperature range, while shell@core clusters can oscillate among the three main nanomorphologies, at least in certain size ranges and at certain temperatures. We hope that this work will stimulate new experimental and theoretical studies to deepen the links between structural stability and the formation/synthesis of metallic nanoalloys.

\section{Acknowledgements}

The authors thank the financial support by the U. K. research council EPSRC, under Grant No. EP/J010812/1 and the financial support offered by the Royal Society under the project number RG120207. KR acknowledges financial support by the U. K. research council EPSRC, Grant No. ER/M506357/1.

\section{References}

1 M. I. Stockman, Science, 2015, 348, 287.

2 G. Barcaro, L. Sementa, A. Fortunelli and M. Stener, Phys. Chem. Chem. Phys., 2015, 17, 27952.

3 V. M. Medel, J. U. Reveles, S. N. Khanna, V. Chauhan, P. Sen and A. W. Castleman, Proc. Natl. Acad. Sci. U. S. A., 2011, 108, 10062.

4 C. Di Paola, R. D'Agosta and F. Baletto, Nano Lett., 2016, 16, 2885.

5 F. Calle-Vallejo, J. Tymoczko, V. Colic, Q. H. Vu, M. D. Pohl, K. Morgenstern, D. Loffreda, P. Sautet, W. Schuhmann and A. S. Bandarenka, Science, 2015, 350, 185.

6 G. G. Asara, L. O. Paz-borbon and F. Baletto, ACS Catal., 2016, 6, 4388.

7 F. Baletto and R. Ferrando, Rev. Mod. Phys., 2005, 77, 371.

8 S. R. Plant, L. Cao and R. E. Palmer, J. Am. Chem. Soc., 2014, 136, 7559.

9 S. Scaramuzza, S. Agnoli and V. Amendola, Phys. Chem. Chem. Phys., 2015, 17, 28076.

10 Z. Swiatkowska-Warkocka, A. Pyatenko, F. Krok, B. R. Jany and M. Marszalek, Sci. Rep., 2015, 5, 9849.

11 Z. W. Wang and R. E. Palmer, Phys. Rev. Lett., 2012, 108, 245502.

12 A. S. Barnard, Nanoscale, 2014, 6, 9983.

13 F. Baletto, J. P. K. Doye and R. Ferrando, Phys. Rev. Lett., 2002, 88, 075503.

14 F. Baletto, A. Rapallo, G. Rossi and R. Ferrando, Phys. Rev. B: Condens. Matter Mater. Phys., 2004, 69, 235421.

15 K. Koga, T. Ikeshoji and K.-i. Sugawara, Phys. Rev. Lett., 2004, 92, 115507.

16 T. Shibata, B. A. Bunker, Z. Zhang, D. Meisel, C. F. Vardeman and J. D. Gezelter, J. Am. Chem. Soc., 2002, 124, 11989.

17 C. Wang, S. Peng, R. Chan and S. Sun, Small, 2009, 5, 567. 18 J. Uppenbrink and D. J. Wales, Faraday Trans., 1991, 87, 215. 19 L. D. Marks, Rep. Prog. Phys., 1994, 57, 603.

20 D. J. Wales and L. J. Munro, J. Phys. Chem., 1996, 100, 2053.

21 B. Cheng and A. H. W. Ngan, J. Chem. Phys., 2013, 138, 164314.
22 Y. K. Lan, C. H. Su, W. H. Sun and A. C. Su, RSC Adv., 2014, 4, 13768.

23 T. Li, S. Lee, S. Han and G. Wang, Phys. Lett. A, 2002, 300, 86.

24 Z. Zhang, W. Hu and S. Xiao, Phys. Rev. B: Condens. Matter Mater. Phys., 2006, 73, 125443.

25 L. Pavan, K. Rossi and F. Baletto, J. Chem. Phys., 2015, 143, 184304.

26 F. Chen and R. L. Johnston, Appl. Phys. Lett., 2008, 92, 023112.

27 A. L. Gould, A. J. Logsdail and C. R. A. Catlow, J. Phys. Chem. $C, 2015,119,23685$.

28 A. L. Gould, K. Rossi, C. R. A. Catlow, F. Baletto and A. J. Logsdail, J. Phys. Chem. Lett., 2016, 7, 4414.

29 W. N. Lipscomb, Science, 1966, 153, 373.

30 A. L. Mackay, Acta Crystallogr., 1962, 15, 916.

31 F. Calvo, A. Fortunelli, F. Negreiros and D. J. Wales, J. Chem. Phys., 2013, 139, 111102.

32 M. Asgari, F. R. Negreiros, L. Sementa, G. Barcaro, H. Behnejad and A. Fortunelli, J. Chem. Phys., 2014, 141, 041108.

33 T. Niiyama, S. Sawada, K. S. Ikeda and Y. Shimizu, Chem. Phys. Lett., 2011, 503, 252.

34 W. He, X. Wu, J. Liu, K. Zhang, W. Chu, L. Feng, X. Hu, W. Zhou and S. Xie, Langmuir, 2010, 26, 4443.

35 D. Bochicchio and R. Ferrando, Eur. Phys. J. D, 2012, 66, 115. 36 R. L. Johnston, L. O. Paz-Borbón, G. Barcaro and A. Fortunelli, J. Chem. Phys., 2008, 128, 134517.

37 K. Laasonen, E. Panizon, D. Bochicchio and R. Ferrando, J. Phys. Chem. C, 2013, 117, 26405.

38 K. Rossi, Y. Soon, L. Pavan and F. Baletto, arXiv:1702.07088, 2017, 813351, [cond-mat.mes-hall].

39 V. Rosato, M. Guillope and B. Legrand, Philos. Mag. A, 1989, 59, 321.

40 D. J. Wales and P. Salamon, Proc. Natl. Acad. Sci. U. S. A., 2014, 111, 617.

41 A. Laio and F. L. Gervasio, Rep. Prog. Phys., 2008, 71, 126601.

42 G. Santarossa, A. Vargas, M. Iannuzzi and A. Baiker, Phys. Rev. B: Condens. Matter Mater. Phys., 2010, 81, 174205.

43 L. Pavan, C. Di Paola and F. Baletto, Eur. Phys. J. D, 2013, 67, 24.

44 J. D. Honeycutt and H. C. Andersen, J. Phys. Chem., 1987, 91, 4950.

45 F. Calvo and C. Mottet, Phys. Rev. B: Condens. Matter Mater. Phys., 2011, 84, 035409.

46 D. Bochicchio and R. Ferrando, Eur. Phys. J. D, 2012, 66, year.

47 G. Rollmann, M. E. Gruner, A. Hucht, R. Meyer, P. Entel, M. L. Tiago and J. R. Chelikowsky, Phys. Rev. Lett., 2007, 99, 083402.

48 C. Mottet, G. Rossi, F. Baletto and R. Ferrando, Phys. Rev. Lett., 2005, 95, 035501.

49 F. Baletto, C. Mottet and R. Ferrando, Phys. Rev. B: Condens. Matter Mater. Phys., 2002, 155420.

50 S. Y. Kim, I.-H. Lee and S. Jun, Phys. Rev. B: Condens. Matter Mater. Phys., 2007, 76, 245407.

51 H. Peng, W. Qi, S. Li and W. Ji, J. Phys. Chem. C, 2015, 119, 2186. 